ERRATA

\title{
Erratum: Consistent integral equations for two- and three-body-force models: Application to a model of silicon [Phys. Rev. E 47, 2491 (1993)]
}

Brian B. Laird, Jun Wang, and A. D. J. Haymet

PACS number(s): 61.20.Gy, 65.50. $+\mathrm{m}, 05.70 . \mathrm{Ce}, 61.25 .-\mathrm{f}, 99.10 .+\mathrm{g}$

Typographical errors occurred in this paper and are corrected below:

Equation (1): The symbol before $\mu N$ should be minus not plus. Also, the equation in the text just above Eq. (1) should be $\Omega=-P V$.

Equation (2): The sum over $i, j$ should be $i<j$; similarly, $i, j, k$ should be $i<j<k$.

Equation (3): $E\left[\left\{g^{(n)}\right\}\right] V$ should be changed to $E\left[\left\{g^{(n)}\right\}\right] / V$.

Equation (3): The integrations are three dimensional; $d r$ should be changed to $d \mathbf{r}$.

Equation (5): The factor $\rho^{-1}$ in the $k$-space integral should be deleted.

Equation (11): The factor $\rho^{2}$ in the first integral should be replaced by the factor $\rho$.

Equation (24): The index $n$ should be replaced by 3 .

Equation (A1): The integrations are three dimensional; $d r$ should be changed to $d \mathbf{r}$.

Equation (A2): The factor $\left(\rho^{\mathcal{B}}\right)^{-1}$ in the $k$-space integral should be deleted.

Figure 5: Due to a programming error in which the final term of Eq. (11) was inadvertently set to zero, Fig. 5 is incorrect. No other results are affected. The correct figure is shown below, together with the correct labels.

We are grateful to Dr. Anna Nyberg for bringing many of these errors to our attention.

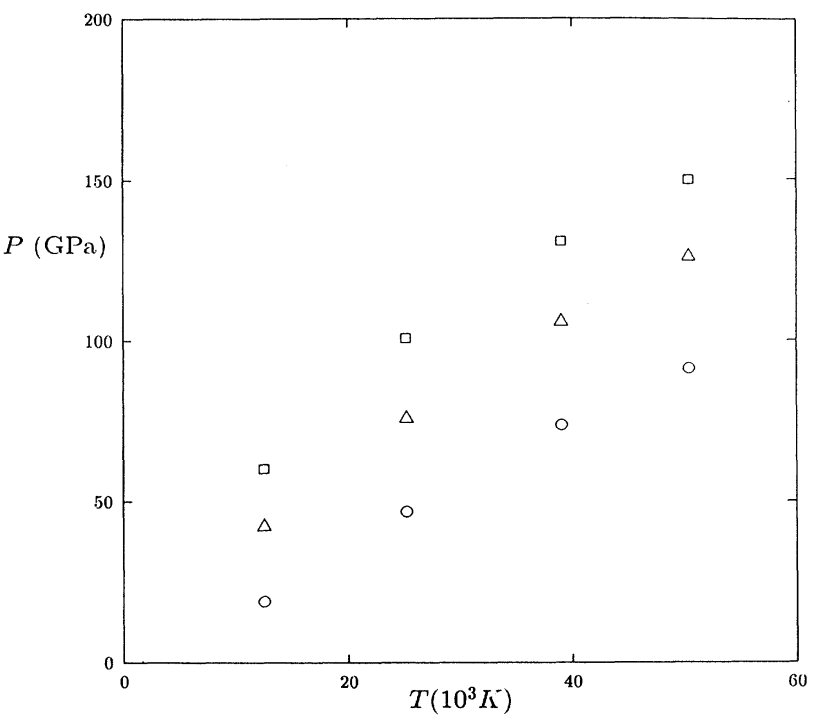

FIG. 5. The predicted pressure for the SW model of silicon at $\rho=0.0525 \AA^{-3}$ as a function of temperature, calculated from the HNC +3 approximation, using both the self-consistent pressure equation (11) (squares) and the virial equation (33) (circles). Also shown for reference is the result from the original figure that sets (incorrectly) the final term of Eq. (11) to zero (triangles). 